Scientific Paper

\title{
Determining the optical properties of blood using He-Ne laser and double integrating sphere set-up
}

\author{
Habib E. ASHOOR ${ }^{1, a}$, Khalil Ebrahim JASIM ${ }^{1}$ \\ ${ }^{1}$ University of Bahrain, Colleges of Science, Department of Physics, Sakheer, P.O.Box:3203, Kingdom of Bahrain \\ ${ }^{a}$ E-mail address: habib.ashoor71@gmail.com
}

(received 28 February 2018; revised 26 November 2018; accepted 5 January 2019)

\begin{abstract}
The behaviour of light interaction with biological tissue is determined by micro-optical parameters: refractive index $(n)$, absorption coefficient $\left(\mu_{a}\right)$, scattering coefficient $\left(\mu_{s}\right)$, and anisotropy $(g)$. The goal of this study is to measure the optical properties of normal whole blood using He-Ne laser (wavelength $632.8 \mathrm{~nm}$ ).

The refractive index is measured using the traveller microscope. The integrating sphere method is used to measure the macro-optical parameters: total diffusive reflectance, transmittance, and collimated transmittance at wavelength 632.8 $\mathrm{nm}$. The macro-optical parameters are fed to Inverse Adding Doubling (IAD) theoretical technique, to estimate the micro-optical parameters $\left(\mu_{s}, \mu_{a}, g\right)$. An alternative practical method is used to measure the $g$ value based on utilising the goniometric table. The study reveals that the refractive index $(n)$ equals $1.395 \pm 0.0547$, absorption coefficient $\left(\mu_{a}\right)$ equals $2.37 \mathrm{~mm}^{-1}$, scattering coefficient $\left(\mu_{s}\right)$ equals $55.69 \mathrm{~mm}^{-1}$, and anisotropy $(\mathrm{g})$ equals 0.82 .

In conclusion, these findings approved, in general, the applicability of the suggested experimental set up. The set up depend on using three devices: the integrating sphere method to estimate $\left(\mu_{s}, \mu_{a}, g\right)$, traveller microscope $(n)$ and goniometer $(g)$.
\end{abstract}

Key words: micro-linear optical properties; whole blood; He-Ne laser; integrating sphere; inverse adding doubling.

\section{Introduction}

The blood of healthy adults is composed of red blood cells (RBCs, 4,500103/L blood), platelets (300103/L blood), white blood cells (WBCs, 8103/L blood), and blood plasma (consists of water, electrolytes, plasma proteins, carbohydrates, lipid and desperate extracellular vesicles). The volume percentage of RBCs in blood is called haematocrit (hct). On average amount, RBCs make up $40 \%$ of the blood found in adult females and $45 \%$ of the blood found in adult males. Haemoglobin is the main component of RBC with concentration of $\pm 350 \mathrm{~g} / \mathrm{L}$ in a cell volume $\pm 90 \mathrm{fL}$ (adult male and female is $140 \mathrm{~g} / \mathrm{L}$ and $155 \mathrm{~g} / \mathrm{L}$ respectively) [1,2]. In non-activated state, RBCs is a discoid cell with diameter 2-4 $\mu \mathrm{m}$ and thickness 0.5-2 $\mu \mathrm{m}$ [3].

In recent years, light has been used in several medical applications. For better results of treatment and clinical diagnostic, the distribution and propagation of light within biological tissues must be determined properly. This can be achieved by measuring the micro-optical parameters of the interested biological tissues.

There are different methods used to describe the distribution and propagation of light in biological medium. However, Radiative Transfer Equation (RTE) is favourable as it only depends on energy; unlike the fundamental transport theory for
Maxwell equations, which is suffer short outcome due to its complex analysis along with the fact that the polarization effect is taken into consideration [4]. Analytically, the general solution for RTE is difficult to derive; hence, simplified models have been used. For instance, Kubelka-Munks [5] approach has been widely used to measure the absorption and scattering coefficients of biological tissues [6-8] that is more applicable for high scatter medium [9]. On the other hand, several authors have used the diffusion approximation of RTE in the analysis of biological samples [10-12]. Recently, two novel methods have been utilized: the Inverse Adding-Doubling (IAD) approach [13] and the Inverse Monte Carlo simulation method $[14,15]$. Although there are several well-established methods used to measure the optical properties of whole blood, further studies are required to develop and suggest novel experimental set up and techniques used to estimate the micro-optical properties more accurately.

In this investigation, the micro-optical properties of the whole blood are measured using double integrating sphere arrangement with $\mathrm{He}-\mathrm{Ne}$ laser $(632.8 \mathrm{~nm})$ as coherent light source. An alternative method of measuring the refractive index, based on using the traveller microscope, is proposed. 


\section{Theoretical background}

The behaviour of light as it propagates in biological tissues is described by integro-differential equation that relates the gradient of the radiance (intensity, $I(\vec{r}, \hat{s}))$, i.e. the travelling of a photon power $\left(\mathrm{Wm}^{-2} \mathrm{sr}^{-1}\right)$, at a position $\vec{r}$ in the direction $\hat{s}$ to attenuate by absorption and scattering, as [16]:

$\hat{s} . \vec{\nabla} I(\vec{r}, \hat{s})+\mu_{t} I(\vec{r}, \hat{s})=\frac{\mu_{s}}{4 \pi} \int_{4 \pi} p\left(\hat{s}, \widehat{s^{\prime}}\right) I(\vec{r}, \hat{s}) d \Omega$

Eq. 1

where $\mu_{t}\left(=\mu_{a}+\mu_{s}\right)$ is the attenuation coefficient in $\mathrm{mm}^{-1}, \mu_{s}$ is the scattering coefficient, $\mu_{a}$ is the absorption coefficient, $d \Omega$ is the differential solid angle about the direction $\hat{s}$, and $p\left(\hat{s}, \widehat{s}^{\prime}\right)$ is the phase function, i.e. probability density function to describe a photon in $\hat{s}$ direction changes to $\widehat{s}^{\prime}$ direction. Usually, the phase function is normalized to unity to provide equal probability direction for the scattered photon.

$\int_{4 \pi} p(\cos (\theta)) d \Omega$

Eq. 2

For biological media, $p(\cos (\theta))$ is normally defined by Henyey-Greenstien phase function $[4,17]$ that is expressed as:

$p(\cos (\theta))=\frac{1}{4 \pi} \frac{1-g^{2}}{\left(1+g^{2}+2 g \cos (\theta)\right)^{\frac{3}{2}}}$

where $\mathrm{g}$ is the anisotropy which is defined as the mean cosine of the scattered photons, expressed as:

$g=\frac{\int_{4 \pi} p(\cos (\theta)) \cos (\theta) d \Omega}{\int_{4 \pi} p(\cos (\theta)) d \Omega}$

The anisotropy has ranges from -1, for complete backward scattering, up to +1 , for complete forward scattering, and is equal to zero for homogeneous scattering, i.e. similar scattering in all directions. Experimentally, the anisotropy is measured by estimating the light intensities at various scattering using a goniometer table. To enhance the detected signal an integrating a convex lens with $10 \mathrm{~mm}$ focal length is fixed on the goniometer table. The $g$ values can be estimated by the average cosine of the scattering angle [6], as:

$g=\frac{\sum_{i} I_{i} \cos \left(\theta_{i}\right)}{\sum_{i} I_{i}}$

the sums are over all $i^{\text {th }}$ of the scattering angle and intensities. Hence, the obtained $g$ values can be compared with those found from IAD technique. Two dimensionless parameters can also been obtained from applying IDA: albedo $(a)$ and the optical depth $(d)$. Albedo is defined as the ratio of the reflected to incident intensities scattering coefficient and which can be found by using the expression [17].

$a=\frac{\mu_{s}}{\mu_{a}+\mu_{s}}$

It approaches a limit of one if the scattering coefficient is dominant, and zero if the absorption coefficient is dominant. The optical length [13] described as the thickness of a sample is expressed as:

$\tau=d\left(\mu_{t}+\mu_{s}\right)$

Eq. 7
The refractive index $(n)$ of the sample and holder plays an essential role on the light path that is measured by a travelling microscope. The application of the travelling microscope depends on comparing the real depth to the apparent depth through the use of the following equation:

$n=\frac{d_{1}-d_{3}}{d_{2}-d_{3}}$

Eq. 8

where $d_{1}-d_{3}$ and $d_{2}-d_{3}$ are the real and apparent depths respectively. The measured $n$ is compared with conventional methods of minimum deviation using an equilateral hollow prism made of thin quartz sides [18]. The advantage of using the traveller microscope is that only a few drops of RBC are required.

To apply IAD method [13], three raw light intensities are required: diffuse reflectance $R_{d}$, total diffuse transmittance $T_{d}$, and collimated transmittance $T_{c}$. The micro-optical parameters, hence, can be found (refer to Table 2).

The anisotropy $g$ value is measured by irradiating the blood sample, placed carefully between two slides glasses, with $\mathrm{He}$ $\mathrm{Ne}$ laser beam $(\lambda=632.8 \mathrm{~nm})$. The scattered intensities, at different angles, were estimated by fixing fiber optics connected with a camera at the edge of a goniometer [6]. The signal at different angles of $5 \mathrm{deg}$ increments from the blood sample is measured through fiber optics, which is connected to a PC to analyze the intensity of the signal.

\section{Experimental}

The blood sample was drawn from a volunteer in the clinical laboratory. The sample is freshly collected into a vacuum tube. The absorption spectrum was taken using Shimadzo spectroscopy at room temperature. The range of absorption spectrum is within the range 200 up to $2000 \mathrm{~nm}$ and peaking at around $430 \mathrm{~nm}$, the absorption band [6].

The refractive index of the diluted whole blood was measured using the travelling microscope. Figure 1 schematic of the experiment setup demonstrates the measurement of the refractive index of the diluted normal RBCs. The refractive index is measured as follows. Place a mark on the base of the blood container and focus on it using travelling microscope; $d_{1}$ reading. Then place the diluted blood sample in the container and focus on the sign viewed through the diluted blood sample; $d_{2}$ reading. Finally, place powder on the top of the blood surface and focus on it; $d_{3}$ reading. Refractive index can be estimated by using Equation 8.

The diffuse reflectance and transmittance of the blood samples are measured by using the double integrating sphere technique (Figure 2). The blood sample was prepared by placing drops of blood between two slides of glasses. Care was taken to avoid undue pressure on the glass slides which may change the optical properties of the blood. Glue was applied all around the glass slides to prevent the dehydration of the blood. The thickness of the sample is estimated using a micrometer, i.e. $0.05 \mathrm{~mm}$. The measurements were repeated three times. 


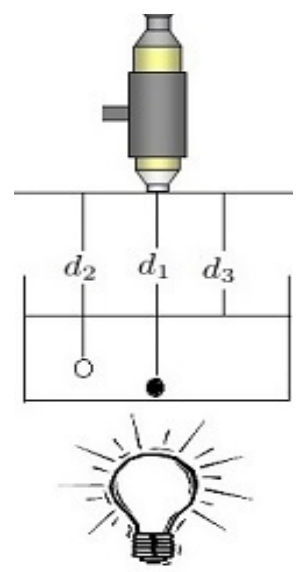

White light

Figure 1. Schematic setup shows traveller microscope used to measure the refractive index of the diluted blood sample. $d_{1}-d_{3}$ is the real depth and $d_{2}-d_{3}$ is the apparent depth. The refractive index is the ratio between the real depth and the apparent depth.

He-Ne (average output power is about $4 \mathrm{~mW}$ having the diameter of $1.25 \mathrm{~mm}$ at $1 / \mathrm{e}^{2}$ and the beam divergence is 0.7 mrad) laser beam was then focused on the sample, i.e. sandwich between the two integrating sphere, using a system of lenses. The integrating sphere (6 inches in diameter) is totally inside coated by barium sulphate. The size of the ports of the two integrating spheres is 1 inch, i.e. diffused transmitted and reflected light intensities measures. A reflecting baffle within the sphere covers the fiber from the direct emission and reflection from the sample. Light is trapped within the two sphere and the transmission and reflection light intensities were detected by the fiber optics that were mounted in the integrating sphere, port.

\section{Results and discussion}

A traveller microscope was used to measure the refractive index of the blood sample (Figure 1). The reflective index is estimated by calculating the ratio between the apparent depths to the real depth. The diluted normal whole blood trial measurements of the concentration of $40 \%$ are recorded in Table 1. From extrapolation of the data, the refractive index of the $100 \%$ normal whole blood was estimated to be about 1.38 . T-students test analysis was performed by using SPSS software. It showed a significant degree of agreement, i.e.t $=4.303$ at $p v a l=0.730$. The sample blood thickness was determined by using the micrometer screw and was measured to be half of the minimum division and is equaled to $0.025 \mathrm{~mm}$.

The total diffuse reflectance and transmittance at $632.8 \mathrm{~nm}$ were determined by placing the blood sample between the two integrated spheres (Figure 2). The collimated was measured by placing the detector $1.5 \mathrm{~m}$ from the transmitted sphere.

These measures were fed IAD approach to estimate the micro-optical properties. As can be seen in Table 3, the value of $\mu_{s}$ is dominant, supporting previous studies found by Steinke

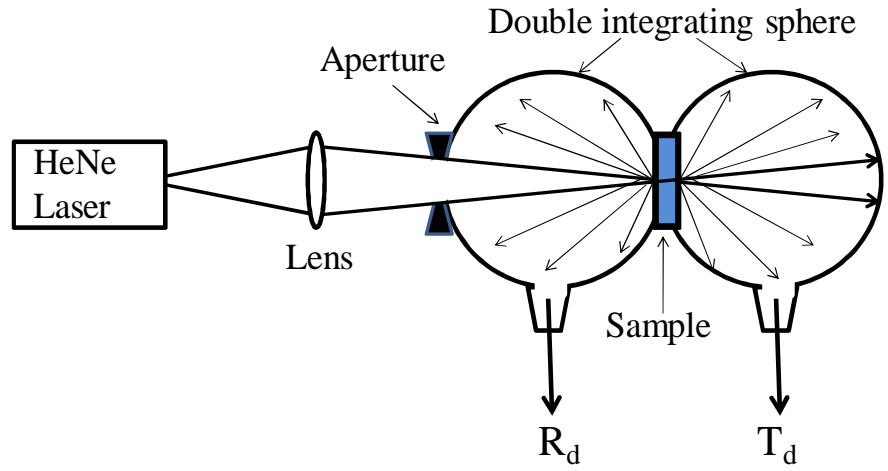

Figure 2. Schematic setup for the diffuse reflectance and transmittance using double integrating spheres.

and Shepherd $[19,20]$ who reported the scatter coefficient of about $100 \mathrm{~cm}^{-1}$.

Furthermore, it is consistent with those Sardar and Levy [6] who found the scattering coefficient to be about $134.4 \mathrm{~cm}^{-1}$ at a wavelength of $488 \mathrm{~nm}$. The higher value of the scattering coefficient could be explained by the fact that most of the whole blood is consists of red blood, i.e. hematocrit (hct). For instance, the scattering coefficient is linearly proportional to the hct and the scattering coefficient saturates for hct $>10 \%$. On the other hand, the resulted $\mu_{\mathrm{a}}$ is disagreeing with Sardar and Levy [6] and this is can be interpreted by the fact that at the wavelength of $488 \mathrm{~nm}, \mathrm{RBC}$ has a high absorption coefficient $[21,22]$.

Table 1. Displays refractive index measures

\begin{tabular}{cccc}
\hline \hline Trial & Real depth & Apparent depth & Refractive index \\
\hline 1 & 6.990 & 5.300 & 1.318 \\
2 & 6.460 & 4.500 & 1.435 \\
3 & 6.450 & 4.500 & 1.433 \\
Average & $6.633 \pm 0.252$ & $4.767 \pm 0.377$ & $1.395 \pm 0.055$ \\
\hline \hline
\end{tabular}

Table 2. Displays the integrating sphere outputs: diffuse reflectance $\left(R_{d}\right)$, the diffuse transmittance $\left(T_{d}\right.$, and collimated transmittance $\left(T_{c}\right)$.

\begin{tabular}{cccc}
\hline \hline$\lambda(\boldsymbol{n m})$ & $\boldsymbol{R}_{\boldsymbol{d}}$ & $\boldsymbol{T}_{\boldsymbol{d}}$ & $\boldsymbol{T}_{\boldsymbol{c}}$ \\
\hline 632.8 & 0.179681 & 0.594469 & 0.048581 \\
\hline \hline
\end{tabular}

Table 3. Displays the IAD outputs: albedo $(a)$, optical length $(\tau)$, the absorption coefficient $\left(\mu_{a}\right)$, scattering coefficient $\left(\mu_{s}\right)$, and anisotropy $(g)$.

\begin{tabular}{ccccc}
\hline \hline $\boldsymbol{a}$ & $\boldsymbol{\tau}$ & $\boldsymbol{\mu}_{\boldsymbol{a}}(\boldsymbol{m m})^{-\boldsymbol{I}}$ & $\boldsymbol{\mu}_{\boldsymbol{s}}(\boldsymbol{m} \boldsymbol{m})^{-\boldsymbol{I}}$ & $\boldsymbol{g}$ \\
\hline 0.96 & 2.90 & 2.37 & 55.69 & 0.82 \\
\hline \hline
\end{tabular}


Table 4. Table showing the He-Ne intensities at the different angle using goniometer device with $5 \mathrm{deg}$ increment. The $\mathrm{g}$ value is calculated using Equation 5.

\begin{tabular}{ccc}
\hline \hline $\boldsymbol{\theta}(\boldsymbol{r a d})$ & $\boldsymbol{I}(\boldsymbol{a} . \boldsymbol{u})$ & $\boldsymbol{I} \times \boldsymbol{c o s}(\boldsymbol{\theta})(\boldsymbol{a} . \boldsymbol{u})$ \\
\hline 0.38 & 64310.10 & 59752.61 \\
0.43 & 15398.30 & 13989.48 \\
0.48 & 19608.60 & 17361.33 \\
0.54 & 255.80 & 219.95 \\
0.59 & 1111.60 & 924.80 \\
0.64 & 16452.20 & 13190.97 \\
0.69 & 15161.40 & 11665.17 \\
0.75 & 11967.30 & 8794.94 \\
0.80 & 6757.90 & 4719.82 \\
0.85 & 1798.00 & 1186.68 \\
0.90 & 692.80 & 429.38 \\
Sum & 153514.00 & 132235.13 \\
\hline
\end{tabular}

Bosschaart et al [16] reported the absorption and scattering coefficients of $2.01 \mathrm{~mm}^{-1}$ and $85.35 \mathrm{~mm}^{-1}$, respectively, at 635 $\mathrm{nm}$. The lower value of the absorption coefficient $\left(3.35 \mathrm{~mm}^{-1}\right)$ was also found by Feng et al [23] using a single integrating sphere and Kubelka-Munk model. Furthermore, Bosschaart et al [16] reported at wavelength $630 \mathrm{~nm}$ that the absorption and scattering coefficients are $2.27 \mathrm{~mm}^{-1}$ and $88.55 \mathrm{~mm}^{-1}$ respectively. The optical depth calculated using equation 7 is almost in agreement with Sardar and Levy [6], who reported the value of $\tau$ to be about 2.13. By comparing the albedo factor measured by this study along with Sardar and Levy [6], the albedo factor is inconsistent. The reason for this is not clear but it may have something to do with the wavelength at which the measurement was taken place. The anisotropy value has a good agreement with Steinke and Shepherd [20] finding, which showed that the anisotropy value to be about 0.98 (Table 3). In addition, our study agreed also with Sardar and Levy [6] who reported the anisotropy value to be about 0.91 . There is an excellent good agreement between the anisotropy obtained using the goniometer and the IAD approach. A similar result was found by Yaroslavsky et al. [24] and Sardar and Levy [6].

The fundamental optical properties of blood discussed in this study are to be of practical significance, and can be implemented in the Radiative Transfer Equation. Through proper suppositions, approximate distribution of light in the blood medium is achievable.

\section{Conclusion}

Interaction of light with a biological sample is described by its micro-optical parameters such as refractive index, scattering and absorption coefficients, and anisotropy. This study set out to determine the micro-optical parameters of blood by using, simple and practical, experimental set up. The set up consists of three main devices: the integrating spheres, travelling microscope, and goniometer. General speaking, the results obtained by the proposed experimental set up are trustworthy and showed an excellent agreement with well-known established set up in the literature. Whilst the suggested experimental set up is reliable, it has some limitations: care must be taken in using the travelling microscope, and the sample thickness and freshness must be considered. The question that needs to be answered about this study is the applicability of this experimental set up in case of using multispectral rang.

\section{Acknowledgment}

The authors would like to express their appreciation to the physics department staff for their kind support. This work is supported by the center of scientific research at the University of Bahrain.

\section{References}

[1] Boulpaep E, Boron F (eds). Medical physiology. $2^{\text {nd }}$ ed. Saunders and Philadephia, PA: Saunders/Elsevier. 2009.

[2] van der Pol E, Boing AN, Harrison P, et al. Classification, functions and clinical relevance of extracellular vesicles. Pharmacol Rev. 2012;64(3):676-705.

[3] Kolesnicova IV, Potapov SV, Yurkin MA, et al. Determination of volume, shape and refractive index of individual blood platelets. J Quant Spectrosc Radiat Transf. 2006;102(1):37-45.

[4] Cheoung WF, Welch A, Prahl S. A Review of the Optical Properties of Biological Tissues. IEEE J Quantum Electron. 1990;26:21662184.

[5] Kubelka P. New contribution to the optics intensly light-scattering materials. J Optical Soc Amer. 1948;38(5):448-457.

[6] Sardar D, Levy L. Optical properties of whole blood. Lasers Med Sci. 1998;13(2):106-111.

[7] van Gemert MJ, Verdaasdounk P, Stassen E, et al. Optical properties of human blood vessel wall and plague. Lasers Surg Med. 1985;5(3):235-237.

[8] Wan S, Anderson RR, Parish JA. Analytical modeling forth optical properties of the skin with within verto and vivo applications. Photochem Photobiol. 1981;34(4):493-449.

[9] Hammer M, Roggan A, Schweitzer D, Muller G. Optical properties of ocular fundus tissues-an in verto study using the doubleintegrating-sphere technique and inverse Monte Carlo simulation. Phys Med Biol. 1995;40(6):963-978. 
[10] Ishimaru A. Wave Propagation and Scattering in Random Media: Vol I. New York: Academic Press; 1978.

[11] Reynolds L, Johnson J, Ishimaru A. Diffuse reflectance from finite blood medium: application to the modeling of fiber optic catheters. Appl Opt. 1978;15(9):2059-2067.

[12] Groenhuis RG, Ferwerda HA, Ten Bosch JJ. Scattering absorption of turbid materials determine from reflection measurements. Appl Opt. 1983;22(1):2456-2467.

[13] Prahl SA, van Gemert MJC, Welch AJ. Determining the optical properties of turbid mediably using the adding-doubling method. Appl Opt. 1993;32(4):559-568.

[14] Hourdakis CJ, Perris A. Monte Carlo estimation of tissue optical properties for use in laser dosimetry. Phys Med Biol. 1995;40(3):351-364.

[15] Jacques S, Wang L. A Monte Carlo modeling for light transpose in tissues. in: Welch AJ, van Gemert MJC (eds.). Optical-thermal response of laser-irradiated tissue. 1995.

[16] Bosschaart N, Edelman G, Aalders M, et al. A literature review and novel theoretical approach on the optical properties of whole blood. Lasers Med Sci. 2014;29(2):453-479.

[17] Prahl S. Light transport in tissue: Univ Texas at Austin; 1988. Ph.D. dissertation.

[18] Grange BW, Stevenson WH, Viskanta R. Reflective index of liquid solutions at low temperature: an accurate measurement. Appl Opt. 1976;15(4):858-859.

[19] Steinke JM, Shepherd AP. Diffusion model of the optical absorbance of whole blood. Opt Soc Am A. 1988;5(6):813-822.

[20] Steinke J, Shepherd AP. Comparison with Mie theory and the light scattering of red blood cells. Appl Opt. 1988;27(19):4027-4033.

[21] Jacques S. Optical properties of biological tissues: a review. Phys Med Biol. 2013;58(14):5007-5008.

[22] Prahl S. Optical Absorption of Hemoglobin. Available at: http://omlc.ogi.edu/spectra/hemoglobin.

[23] Feng S, Liu L, Chen R, et al. Scattering and Absorbing Characteristics of Human Whole Blood in K-M Model at He-Ne Laser in Virto. J Fujian Normal Univ (Natural Science). 2003;19(4):46-94.

[24] Yaroslavsky A, Yaroslavsky I, Goldbach T, Schwarzmaier IJ. Influence of the scattering phase function approximation on the optical properties of blood determined from the integrating sphere measurements. J Biomed Opt. 1999;4(1):47-53. 